\title{
Analysis of the Effects of Breast Reconstruction in Breast Cancer Patients Receiving Radiotherapy after Mastectomy
}

\author{
Seong Hwan Kim, Jeong Min Kim, Sun Hyung Park, Sam Yong Lee \\ Department of Plastic and Reconstructive Surgery, Chonnam National University Hospital, Chonnam National University School of \\ Medicine, Gwangju, Korea
}

Background Immediate breast reconstruction after mastectomy and delayed breast reconstruction with post-supplementary treatment are the two types of breast reconstruction currently performed when treating breast cancer. Post-mastectomy radiation therapy (PMRT) not only reduces local recurrence but also improves overall survival. However, the complications and survival rates associated with PMRT need to be clear when determining the timing of breast reconstruction. Accordingly, we investigated the optimal timing of breast reconstruction by observing patients who underwent mastectomy followed by PMRT, based on their overall health and aesthetic satisfaction.

Methods We retrospectively reviewed 21 patients who underwent breast reconstruction with PMRT between November 2004 and November 2010. We collected data regarding the various methods of mastectomy, and the modality of adjuvant therapy, such as chemotherapy, hormone therapy, and radiotherapy. Telephone interviews were conducted to study the general and aesthetic satisfaction.

Results Patients who received PMRT after breast reconstruction showed a greater complication rate than those undergoing breast reconstruction after PMRT $(P=0.02)$. Aesthetic satisfaction was significantly higher in the groups undergoing breast reconstruction after PMRT $(P=0.03)$. Patients who underwent breast reconstruction before PMRT developed complications more frequently, but they expressed greater aesthetic satisfaction with the treatment.

Conclusions It is recommended that the complication rates and aesthetic satisfaction after breast reconstruction be carefully considered when determining the optimal timing for radiotherapy.

Keywords Breast / Mastectomy / Radiotherapy

\author{
Correspondence: Sam Yong Lee \\ Department of Plastic and \\ Reconstructive Surgery, Chonnam \\ National University School of \\ Medicine, 42 Jebong-ro, Dong-gu, \\ Gwangju 501-757, Korea \\ Tel: +82-62-220-6363 \\ Fax: +82-62-227-1639 \\ E-mail: sylee@chonnam.ac.kr
}

No potential conflict of interest relevant to this article was reported.

Received: 20 Jan 2012 • Revised: 15 Feb 2012 • Accepted: 26 Feb 2012

pISSN: 2234-6163 • elSSN: 2234-6171 • http://dx.doi.org/10.5999/aps.2012.39.3.222• Arch Plast Surg 2012;39:222-226

\section{INTRODUCTION}

The prevalence of breast cancer is increasing in Korea and in the West. In 2008, breast cancer was the second most frequent cancer affecting women in Korea [1]. The increased incidence of breast cancer can be explained by an increasingly Westernized lifestyle, a decrease in child birth and lactation, and early breast cancer screening. However, early detection, surgery, chemotherapy, radiotherapy, and hormone therapy have reduced the mortality rate of breast cancer [2]. In particular, for mastectomy and 
radiation therapy, reductions in local recurrence and improvements in overall survival have been demonstrated in many studies. However, with post-mastectomy radiation therapy (PMRT), it is difficult to determine the optimal timing of radiation therapy in relation to breast reconstruction. Immediate reconstruction and delayed reconstruction have both been performed. Immediate reconstruction has many advantages over delayed reconstruction and is currently preferred [3]. However, delayed reconstruction is preferred in cases requiring radiation therapy due to the complications of PMRT. In addition, factors such as aesthetics and patient satisfaction play a major role in determining the sequence and timing of PMRT and breast reconstruction $[4,5]$.

In this paper, we study the timing of breast reconstruction for patients who have undergone mastectomy with radiation therapy, that is, PMRT before and after breast reconstruction, focusing on complications, overall health, and aesthetic satisfaction.

\section{METHODS}

\section{Patients}

We retrospectively studied 21 patients who underwent mastectomy as well as PMRT as adjuvant therapy for breast cancer from November 2004 to November 2010. The patients were divided into 2 groups: 1) the group that received breast reconstruction immediately after mastectomy, followed by radiation therapy, and 2) the group that received delayed breast reconstruction following PMRT. The mean periods between radiotherapy and breast reconstruction were 1.2 months and 7.1 months in the former and the latter groups, respectively.

\section{Methods}

A retrospective study on mastectomy, PMRT, chemotherapy, and hormone therapy was conducted using medical records, including cases in which complications had occurred during treatment. Surgical complications included systemic complications such as recurrence, metastasis, flap necrosis, fat necrosis, hematoma, and local complications.

The patients' overall condition and aesthetic satisfaction in the long-term follow-up were assessed via a telephone survey. The telephone survey was performed a second time after a 4-month interval. The Michigan Breast Reconstruction Outcome Study questionnaire contained the following 5 items about overall satisfaction: 1) Knowing what I know today, I would choose to have breast reconstruction. 2) Knowing what I know today, I would choose to have the type of reconstruction I had. 3) Overall, I am satisfied with my reconstruction. 4) I would recommend the type of reconstructive procedure that I had to a friend. 5) I feel I had sufficient information regarding the procedure to make an informed choice. With regard to aesthetic satisfaction, the following 2 items were used: 1) The size and shape of my breasts are the same. 2) It feels natural and tender when I touch my breasts. Questionnaire responses were scored on a 5-point Likert scale with scores ranging from "very satisfied" 5) to "very dissatisfied" 1).

\section{Statistical analysis}

For statistical analysis, SPSS ver. 17.0 (SPSS Inc., Chicago, IL, USA) was used. For the implementation time and complications of the radiation therapy as well as the overall satisfaction and aesthetic satisfaction, the chi-square test was used. The internal consistency of the questionnaire was assessed using Cronbach's alpha. The test-retest reliability was analyzed using Pearson's correlation coefficient. $\mathrm{P}<0.05$ was defined as statistically significant.

\section{RESULTS}

\section{Patient characteristics}

The average age of the patients was 40.8 years. The patients who received radiation therapy after breast reconstruction averaged 36.3 years of age, whereas the group that received breast reconstruction after radiation averaged 48.0 years of age. None of the 21 patients had notable medical histories. Among them, 15 patients underwent a modified radical mastectomy for breast cancer, 3 patients underwent skin-sparing mastectomy with axillary lymph node dissection, and 3 patients underwent nipple-sparing mastectomy with axillary lymph node dissection. Two patients had stage 0 cancer, 3 patients had stage 1,8 patients had stage 2 , 6 patients had stage 3 , and 2 patients had stage 4 cancer. In total, 16 patients received adjuvant chemotherapy, 13 received hormone therapy, and 21 received radiation therapy. The average hospital stay for the breast reconstruction patients was 21.7 days. The hospital stay after PMRT was 17.1 days on average (Table 1). Transverse rectus abdominis myocutaneous (TRAM) flap breast reconstruction was performed in 15 patients, a latissimus dorsi musculocutaneous island flap was performed in 4 patients, and an extended latissimus dorsi musculocutaneous island flap was performed in 2 patients.

Table 1. Patient demographics

\begin{tabular}{|lcc|}
\hline & $\begin{array}{c}\text { Reconstructionbefore } \\
\text { PMRT }(\mathbf{n = 1 3})\end{array}$ & $\begin{array}{c}\text { Reconstruction after } \\
\text { PMRT }(\mathbf{n = 8})\end{array}$ \\
\hline Age & $36.3 \pm 6.0$ & $48.0 \pm 3.3$ \\
Chemotherapy & $11 / 13(84.6)$ & $5 / 8(62.5)$ \\
Hormone therapy & $10 / 13(76.9)$ & $3 / 8(37.5)$ \\
Hospital days & $21.7 \pm 9.4$ & $17.1 \pm 4.7$ \\
\hline $\begin{array}{l}\text { Values are presented as mean } \pm \text { standard deviation or number }(\%) . \\
\text { PMRT, postmastectomy radiotherapy. }\end{array}$ \\
\hline
\end{tabular}


Complications that occurred after breast reconstruction included seroma, hematoma, contracture, fat necrosis, and flap loss. In total, 6 cases of complications were reported for the group that underwent breast reconstruction before radiation therapy, whereas only 1 case was reported for the group that underwent breast reconstruction after radiation therapy. Therefore, the incidence of complications in the group receiving PMRT after breast reconstruction was significantly higher than that of the group receiving PMRT before breast reconstruction $(\mathrm{P}=0.03)$ (Table 2). The mean dosage of radiation therapy in the groups for breast reconstruction before and after PMRT was 5,632.3 cGy and 5,837.5 cGy, respectively. The difference in the dosage between the two groups was statistically insignificant.

In the breast reconstruction before PMRT group that received hormone therapy, the complication rate was $6 / 10(0.6)$. The complication rate of patients who did not receive hormone therapy in the aforementioned group was $2 / 3(0.66)$. In the group that had breast reconstruction after PMRT, the patients who received hormone therapy had no complications, where as the patients who did not receive hormone therapy showed a complication rate of $1 / 3(0.33)$. The complication rate based on having received hormone therapy was statistically insignificant.

\section{Findings from the questionnaire}

The patients' overall satisfaction with breast reconstruction was 22.3 on a scale of 1-25 after radiation therapy and 22.2 for PMRT after breast reconstruction; there was no significant difference between these groups. With regard to aesthetic satisfaction after breast reconstruction, the patients who underwent PMRT after surgery had an average score of 8.3 on the Likert scale, and this score was 7.0 for the group that underwent breast reconstruction after radiation therapy; the difference was found to be statistically significant $(\mathrm{P}=0.03)$ (Table 3 ).

The Cronbach's alpha assessing the internal consistency of the questionnaire regarding the patient's overall satisfaction and aesthetic satisfaction was 0.787 and 0.755 , respectively. Test-retest reliability was assessed with Pearson's correlation coefficient

\section{Table 2. Complications}

\begin{tabular}{|lcc|}
\hline & $\begin{array}{c}\text { Reconstruction } \\
\text { before PMRT }\end{array}$ & $\begin{array}{c}\text { Reconstruction } \\
\text { after PMRT }\end{array}$ \\
\hline Seroma & 2 & 0 \\
Hematoma & 0 & 1 \\
Contracture & 1 & 0 \\
Fat necrosis & 1 & 0 \\
Flap loss & 2 & 0 \\
Total incidence & 6 & 1 \\
\hline PMRT, postmastectomy radiotherapy. & \\
\hline
\end{tabular}

$(\mathrm{P}<0.001)$.

\section{DISCUSSION}

Classical mastectomy, radiation therapy, and chemotherapy are currently used to treat breast cancer. A $10 \%$ increase in survival has been reported in patients with lymph-node-positive breast cancer undergoing PMRT [2,6]. Accordingly, radiation therapy after breast cancer treatment has been increasingly implemented, but the appropriate timing of breast reconstruction and PMRT remains of great concern [7].

Radiation therapy before breast reconstruction is preferable because the appropriate amount of radiation can be applied following mastectomy; it is difficult to deliver sufficient radiation to the target when breast reconstruction flaps, tissue expanders, or implants are present as a result of breast reconstruction [8]. Thus, the presence of reconstructed tissue or implants mitigates the effects of PMRT, thereby requiring an increase in radiation during therapy, which can cause dermatitis. In addition, it is more difficult to precisely target the tumor tissue; therefore, the radiation can damage the heart, lungs, or other organs in close proximity to the breast with possibly fatal consequences. In this study, the high rate of complications associated with PMRT administered after breast reconstruction suggests that the radiation is not precisely delivered to the target and/or that the amount is not sufficient for treatment (Table 2). Thus, it is preferable to perform radiation therapy before breast reconstruction.

Radiation permanently damages the microvasculature and can lead to fibrosis and failure of the wound healing process [9]. Evans et al. [10] studied 39 cases of implant-based breast reconstructions with radiation treatment and 338 cases of implants without radiation therapy; they reported significantly increased capsular contracture, pain, implant removal, and complications in the group receiving PMRT. Spear and Onyewu [11] suggested

Table 3. Patient satisfaction

\begin{tabular}{|lcc|}
\hline & $\begin{array}{c}\text { Reconstruction } \\
\text { before PMRT }\end{array}$ & $\begin{array}{c}\text { Reconstruction } \\
\text { after PMRT }\end{array}$ \\
\hline General satisfaction & $22.3 \pm 1.2$ & $22.2 \pm 1.2$ \\
Q1 & $4.4 \pm 0.5$ & $4.5 \pm 0.5$ \\
Q3 & $4.3 \pm 0.8$ & $4.7 \pm 0.4$ \\
Q4 & $4.6 \pm 0.4$ & $4.5 \pm 0.5$ \\
Q5 & $4.4 \pm 0.5$ & $4.2 \pm 0.4$ \\
Aesthetic satisfaction & $4.3 \pm 0.6$ & $4.2 \pm 0.4$ \\
\hline $\begin{array}{l}\text { PMRT, postmastectomy radiotherapy; Q1, knowing what I know today, I would } \\
\text { choose to have breast reconstruction; Q2, knowing what I know today, I would } \\
\text { choose to have the type of reconstruction I had; Q3, overall, I am satisfied with my } \\
\text { reconstruction; Q4, I would recommend the type of reconstructive procedure that I } \\
\text { had to a friend; Q5, I feel I had sufficient information regarding the procedure to } \\
\text { make an informed choice. }\end{array}$ \\
\hline
\end{tabular}


the use of a non-autologous flap, but the TRAM flap of Tran et al. [12] and the deep inferior epigastric perforator flap of Rogers and Allen [13] were associated with decreased aesthetic satisfaction as well as flap fibrosis, fat necrosis, flap contracture, and asymmetry of the breast resulting from volume loss. Fosnot et al. [14] reported an increase in flap revision caused by blood vessels of the irradiated flaps. The present study similarly found a higher rate of complications in the group that underwent breast reconstruction after PMRT (Table 2). The present study evaluated various types of reconstructed flaps including the TRAM flap, the latissimus dorsi musculocutaneous island flap, and the extended latissimus dorsi musculocutaneous island flap, and no significant differences in the complication rates were observed among the different flap types. Therefore, radiation therapy generally provides a lower complication rate.

In addition, in this paper, metastasis occurred in patients receiving PMRT after breast reconstruction, which suggests that death may result from margin-positive cancer, lymph node involvement, or micro-metastasis that remains after mastectomy in delayed reconstruction. Buchholz et al. [15] reported an increase in the rate of metastasis and a reduction in the survival rate of patients who needed radiation therapy, but delayed treatment for 6 months. Thus, reconstruction is performed immediately after mastectomy to shorten the treatment period and allow for faster commencement of radiation therapy.

In the present study, complications occurred more frequently with early breast reconstruction. However, the patients receiving early breast reconstruction expressed greater aesthetic satisfaction than the patients receiving radiation therapy and delayed breast reconstruction (Fig. 1). Similar findings were also reported by Khansa et al. [16] and Lee et al. [17]. Breast reconstruc-

\section{Fig. 1. Percent patient satisfaction}

There was no difference in the general satisfaction of the two groups. Aesthetic satisfaction was significantly greater in the reconstruction before postmastectomy radiotherapy (PMRT) group.

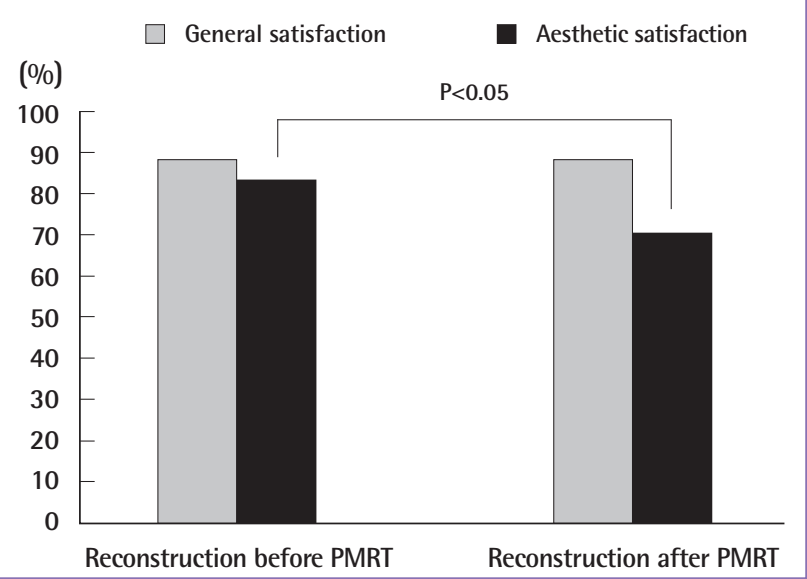

tion compensates for the emotional loss of patients undergoing mastectomy and provides support for the patient's self-image [18-22]. The incidence of depression was found to be higher in patients undergoing delayed reconstruction, and sexual desire and satisfaction were also reduced in the same group [19]. Patients receiving immediate reconstruction have a shorter overall treatment period, which reduces the cost of treatment $[23,24]$. Thus, when determining the appropriate timing for breast reconstruction, medical complications as well as the patient's psychological condition and satisfaction should be taken into account.

The treatment of breast cancer is no longer confined to one aspect of the medical field; it should include physicians, surgeons, radiologists, and plastic surgeons, and it should also address the patients' own opinions and goals. The limitations of this study are as follows. First, the study was a retrospective investigation that evaluated patients between 1 and 7 years after treatment, which decreased the consistency of the results. Second, although the response rate of the telephone survey was $100 \%$, the exclusion of the surgeons' opinion of the overall procedure may have also affected the results. This study evaluated the timing of breast reconstruction and PMRT for breast cancer with respect to aesthetic satisfaction and complications. The patient's satisfaction, complications, and reduction of metastasis are essential factors in the decision-making process for breast reconstruction and thus require further intensive study.

\section{REFERENCES}

1. Park SK, Kim Y, Kang D, et al. Risk factors and control strategies for the rapidly rising rate of breast cancer in Korea. J Breast Cancer 2011;14:79-87.

2. Clarke M, Collins R, Darby S, et al. Effects of radiotherapy and of differences in the extent of surgery for early breast cancer on local recurrence and 15-year survival: an overview of the randomised trials. Lancet 2005;366:2087-106.

3. Han SH, Cho JH, Ahn SH. Immediate breast reconstruction after mastectomy. J Korean Soc Plast Reconstr Surg 1995;22:843-51.

4. Andrade WN, Baxter N, Semple JL. Clinical determinants of patient satisfaction with breast reconstruction. Plast Reconstr Surg 2001;107:46-54.

5. Alderman AK, Wilkins EG, Lowery JC, et al. Determinants of patient satisfaction in postmastectomy breast reconstruction. Plast Reconstr Surg 2000;106:769-76.

6. Overgaard M, Hansen PS, Overgaard J, et al. Postoperative radiotherapy in high-risk premenopausal women with breast cancer who receive adjuvant chemotherapy. Danish 
Breast Cancer Cooperative Group 82b Trial. N Engl J Med 1997;337:949-55.

7. Recht A, Edge SB, Solin LJ, et al. Postmastectomy radiotherapy: clinical practice guidelines of the American Society of Clinical Oncology. J Clin Oncol 2001;19:1539-69.

8. Motwani SB, Strom EA, Schechter NR, et al. The impact of immediate breast reconstruction on the technical delivery of postmastectomy radiotherapy. Int J Radiat Oncol Biol Phys 2006;66:76-82.

9. Jolles B, Harrison RG. Enzymic processes and vascular changes in the skin radiation reaction. Br J Radiol 1966;39: 12-8.

10. Evans GR, Schusterman MA, Kroll SS, et al. Reconstruction and the radiated breast: is there a role for implants? Plast Reconstr Surg 1995;96:1111-5.

11. Spear SL, Onyewu C. Staged breast reconstruction with saline-filled implants in the irradiated breast: recent trends and therapeutic implications. Plast Reconstr Surg 2000;105: 930-42.

12. Tran NV, Chang DW, Gupta A, et al. Comparison of immediate and delayed free TRAM flap breast reconstruction in patients receiving postmastectomy radiation therapy. Plast Reconstr Surg 2001;108:78-82.

13. Rogers NE, Allen RJ. Radiation effects on breast reconstruction with the deep inferior epigastric perforator flap. Plast Reconstr Surg 2002;109:1919-24.

14. Fosnot J, Fischer JP, Smartt JM Jr, et al. Does previous chest wall irradiation increase vascular complications in free autologous breast reconstruction? Plast Reconstr Surg 2011; 127:496-504.

15. Buchholz TA, Austin-Seymour MM, Moe RE, et al. Effect of delay in radiation in the combined modality treatment of breast cancer. Int J Radiat Oncol Biol Phys 1993;26:23-35.

16. Khansa I, Colakoglu S, Curtis MS, et al. Postmastectomy breast reconstruction after previous lumpectomy and radiation therapy: analysis of complications and satisfaction. Ann Plast Surg 2011;66:444-51.

17. Lee BT, T AA, Colakoglu S, et al. Postmastectomy radiation therapy and breast reconstruction: an analysis of complications and patient satisfaction. Ann Plast Surg 2010;64:679-83.

18. Yurek D, Farrar W, Andersen BL. Breast cancer surgery: comparing surgical groups and determining individual differences in postoperative sexuality and body change stress. J Consult Clin Psychol 2000;68:697-709.

19. Dorval M, Maunsell E, Deschenes L, et al. Type of mastectomy and quality of life for long term breast carcinoma survivors. Cancer 1998;83:2130-8.

20. Al-Ghazal SK, Sully L, Fallowfield L, et al. The psychological impact of immediate rather than delayed breast reconstruction. Eur J Surg Oncol 2000;26:17-9.

21. Schain WS, Wellisch DK, Pasnau RO, et al. The sooner the better: a study of psychological factors in women undergoing immediate versus delayed breast reconstruction. Am J Psychiatry 1985; 142:40-6.

22. Handel N, Silverstein MJ, Waisman E, et al. Reasons why mastectomy patients do not have breast reconstruction. Plast Reconstr Surg 1990;86:1118-22.

23. Elkowitz A, Colen S, Slavin S, et al. Various methods of breast reconstruction after mastectomy: an economic comparison. Plast Reconstr Surg 1993;92:77-83.

24. Khoo A, Kroll SS, Reece GP, et al. A comparison of resource costs of immediate and delayed breast reconstruction. Plast Reconstr Surg 1998;101:964-8. 